\title{
A new view of the beta cell
}

\author{
J. Ahnfelt-Rønne • J. Hecksher-Sørensen • L. Schäffer • \\ O. D. Madsen
}

Received: 1 May 2012 / Accepted: 3 May 2012 /Published online: 18 July 2012

(C) Springer-Verlag 2012

Keywords Alpha cell $\cdot$ Beta cell $\cdot$ Fluorescent probe . Functional-mass $\cdot$ Homing $\cdot$ Imaging $\cdot$ Internalisation $\cdot$ Islet of Langerhans

$\begin{array}{ll}\text { Abbreviations } \\ \text { DTBZ } & \text { Dihydrotetrabenazine } \\ \text { GLP-1 } & \text { Glucagon-like peptide 1 } \\ \text { PET } & \text { Positron emission tomography } \\ \text { TMEM27 } & \text { Transmembrane protein 27 }\end{array}$

There is a current unmet need in diabetology for a direct measure of the functional beta cell mass in patients, partly as a result of a lack of appropriate probes. In this issue of Diabetologia, Vats and colleagues describe the generation of a new antibody raised against transmembrane protein 27 (TMEM27) with promising features for use in beta cell imaging in humans [1].

Diabetes mellitus is characterised by high blood glucose levels caused by an insufficient functional beta cell mass, which is determined by the actual beta cell mass and how much

\section{J. Ahnfelt-Rønne • O. D. Madsen $(\bowtie)$}

Islet Biology, Hagedorn Research Institute,

Niels Steensensvej 1,

DK-2820 Gentofte, Denmark

e-mail: odm@hagedorn.dk

J. Hecksher-Sørensen

Imaging Team, Histology and Delivery, Novo Nordisk A/S,

Måløv, Denmark

\section{Schäffer}

Diabetes Protein Engineering, Novo Nordisk A/S,

Måløv, Denmark

J. Ahnfelt-Rønne · J. Hecksher-Sørensen • L. Schäffer •

O. D. Madsen

Novo Nordisk A/S,

Måløv, Denmark insulin the beta cells produce relative to the physiological demand. Diabetes is a group of metabolic diseases with completely different aetiologies; there are three main types: type 1 diabetes is caused by autoimmune destruction of the beta cells, resulting in an absolute beta cell deficiency, whereas type 2 diabetes and gestational diabetes are caused by a relative beta cell deficiency resulting from peripheral insulin resistance and inadequate beta cell function, which is followed by loss of beta cell mass in type 2 diabetes.

The beta cell mass is plastic, allowing adaptation to physiological demand. For example, the pancreas is able to increase the beta cell mass during pregnancy and in obesity, at least in rodents, thus compensating for the increased demand for insulin caused by insulin resistance. Diabetes develops when this mechanism fails, and it is therefore thought that drugs that improve the capacity to expand the beta cell mass or, alternatively, prevent the loss of beta cell mass will be of great clinical importance in the treatment of diabetes. Evidence from mouse studies has suggested that, even after near complete loss of beta cells, the pancreas has the capacity to spontaneously regenerate a sufficiently large functional beta cell mass to become independent of insulin treatment [2]. Furthermore, measurable levels of circulating C-peptide, as well as pancreatic insulin-positive cells, can be found in type 1 diabetic patients, even many years after the onset of the disease [3]. This is indicative of sustained formation of new beta cells, lending hope to the notion that induction of immune tolerance could offer a potential cure for type 1 diabetes, perhaps in combination with regenerative medicine.

Although beta cell mass declines in both type 1 and type 2 diabetes in humans, the process is poorly understood because only indirect means are available for estimation of the beta cell mass in vivo. These are based on correlations between functional insulin secretion measurements and beta cell mass [4]. Furthermore, establishment of the beta cell mass by morphometry on biopsies may be grossly misleading owing to 
the heterogeneity of islet distribution in the organ. Thus, although biopsies have greatly facilitated the study of disease progression in organs such as the skin and the kidneys, this approach is less attractive in diabetes.

Alternative robust methods for determining the beta cell mass are therefore required in order to study the onset and progression of the disease, providing endpoints in clinical trials and measurements of the efficacy of novel therapies aimed at the preservation or regeneration of beta cells. It is interesting to note that animal studies have suggested that drugs that increase the level of glucagon-like peptide 1 (GLP-1) signalling may in part act through a positive effect on beta cell mass [5]. Several such drugs are on the market for the treatment of diabetes, but it is unknown whether they modulate beta cell mass in humans.

In vivo imaging of the pancreas and the beta cells contained therein could potentially allow the direct measurement of beta cell mass. However, although great progress has been made in medical imaging, in the form of magnetic resonance and positron emission tomography, imaging beta cells has proved to be a tremendous challenge. An inherent problem in beta cell imaging is their rarity and heterogeneous distribution in small (submillimetre) clusters throughout the pancreas, where they only constitute about $1-2 \%$ of the total organ mass.

No currently available method has enough spatial resolution to image native islets in vivo and it is therefore critical to identify probes that will increase islet contrast and produce a signal proportional to islet mass. The discovery that beta cells express vesicular monoamine transporter type 2 (VMAT2) has prompted researchers to use one of its ligands, dihydrotetrabenazine (DTBZ), for beta cell imaging [6]. DTBZ is used clinically for positron emission tomography (PET) imaging of dopaminergic neurons in the central nervous system and is the only tracer currently being clinical trialled for beta cell imaging by PET [7]. However, recent multimodal imaging has provided strong evidence that this approach may have limited use for beta cell imaging because the PET signal generated does not correlate with beta cell mass [8]. In their paper [8] the authors stipulate important directions for future preclinical evaluations of beta cell probes.

A similar approach has recently demonstrated promising results for in vivo optical imaging in mice. The GLP-1 receptor agonist exendin- 4 can be fluorescently conjugated and injected intravenously in mice. Owing to high levels of the GLP-1 receptor on beta cells, the probe internalises and accumulates rapidly in the beta cells, allowing in vivo monitoring of the fluorescent signal which, importantly, shows a good correlation with beta cell mass determined by stereology. Furthermore, the probe effectively demonstrates loss of beta cell mass following streptozotocin-induced diabetes $[9,10]$. We have generated similar probes and have likewise observed the rapid accumulation of these in beta cells in vivo. In addition, we have demonstrated their potential for beta cell imaging by using optical projection tomography on isolated pancreases (Fig. 1). Although optical imaging is of limited use for total pancreas imaging in humans, the demonstration of very high probe accumulation and good signal-to-background ratios indicate that further investigation of this probe for use in PET imaging is warranted.

An alternative to the use of receptor/ligand pairs for probing beta cells is to use antibodies with beta cell surface reactivity and specificity. A monoclonal antibody with these characteristics (recognising an uncharacterised antigen, IC2) was generated in 1986 and was subsequently used to probe beta cells in vivo, although the analyses of specificity and correlation with beta cell mass were performed in isolated pancreases $[11,12]$. The fact that this antibody is a large IgM class antibody and has a long plasma half-life is likely to reduce its application in human beta cell imaging owing to a poor signal-to-noise ratio [7]

Promising probes were recently generated by panning a phage display in vivo and in vitro [13]. A single-chain antibody was identified that efficiently labels beta cells in vivo, and radiolabelling demonstrated a good correlation with beta cell mass in the isolated organ. The probe is cleared rapidly from the bloodstream and, although it was identified in a screen in rodents, the antibody also exhibits specific in situ binding to human islets. Interestingly, the antibody is internalised by beta cells. This feature is common to IC2 and exendin-4 probes and may represent an important feature required to generate sufficient label retention by target cells for imaging.

In this issue of Diabetologia, Vats and colleagues [1] present a novel monoclonal antibody directed against human transmembrane protein 27 (TMEM27). TMEM27 has previously been described as a beta cell marker and proposed to be a regulator of beta cell proliferation [14, 15]. Vats et al describe the first successful generation of
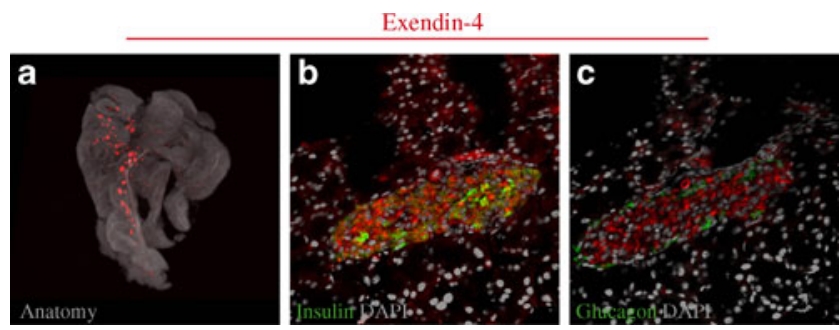

Fig. 1 Mice were injected with 4 nmol of probe (exendin 4 conjugated to Alexa Fluor 594) through the tail vein. The pancreases were harvested after $2 \mathrm{~h}$ and processed for imaging. (a) The splenic lobe of the pancreas imaged by optical projection tomography [18]. The islet signal derived from the probe is shown in red and the anatomy projected from autofluorescence is grey. (b, c) A section from a probed pancreas co-stained for insulin and glucagon using indirect immunohistochemistry. Note the yellow appearance of the beta cells in (b) resulting from colabelling by the probe and the insulin antibody. The alpha cells are not labelled, as demonstrated by co-staining with a glucagon antibody (c) 
an antibody to a known antigen on the beta cell that has properties allowing the in vivo imaging of beta cells. On human tissue arrays containing samples of a range of abdominal organs, the new antibody specifically labelled beta cells in the pancreas and also labelled cells in the kidney, but it did not cross-react with mouse TMEM27. The authors provide evidence that INS-1E cells overexpressing human TMEM27 can be imaged with the antibody conjugated with a near-infrared fluorophore when grafted subcutaneously in nude mice. In similar subcutaneous grafts, specific radioactive uptake following i.v. administration of a radiolabelled probe could clearly be imaged by PET. More importantly, the authors were able to demonstrate that the antibody specifically targets beta cells in vivo and that it gets internalised into the beta cells, possibly allowing a better accumulation of the signal. This was demonstrated by the administration of the antibody coupled to a nearinfrared conjugate in mice in which human TMEM27 was expressed under the control of the rat insulin promoter.

Taken together, this probe seems to offer an attractive new reagent for the in vivo determination of beta cell mass. However, some issues need to be addressed to truly demonstrate its potential. Since the antibody does not crossreact with mouse TMEM27, all studies were conducted using transgenic expression of the human protein. The expression levels were determined to be significantly higher than endogenous expression in human islets, and it will be important to demonstrate signal-to-noise ratios at endogenous expression levels of the target. Also, the lack of crossreactivity with the mouse protein prevented the generation of a signal in the kidneys of the transgenic mice; however, this could potentially be a problem in a clinical setting. It would be interesting to further characterise this antibody to demonstrate correlation with beta cell mass. Grafting human islets in mice is another potential way of demonstrating beta cell labelling at TMEM27 levels closer to normal. We and others have recently reported the finding and characterisation of cell surface reactive antibodies with beta cell specificity in the pancreas $[16,17]$. A combination of existing probes (e.g. TMEM27 antibodies with GLP-1 receptor agonists) and novel, yet to be characterised, probes that use complementary internalisation mechanisms, could potentially improve the sensitivity in medical imaging methods.

Acknowledgements The work leading to Fig. 1 has received support from the Innovative Medicines Initiative Joint Undertaking under grant agreement no. 155005 (IMIDIA), resources of which are composed of financial contributions from the European Union Seventh Framework Programme (FP7/2007-2013) and the European Federation of Pharmaceutical Industries and Associations (EFPIA) companies.

Duality of interest The authors are employed by and own employeegranted shares in Novo Nordisk.
Contribution statement All authors were responsible for the conception and design of the manuscript, drafting the article and revising it critically for important intellectual content. All authors approved the version to be published.

\section{References}

1. Vats D, Wang H, Esterhazy D (2012) Multimodal imaging of pancreatic beta cells in vivo by targeting the cell surface protein, transmembrane protein 27 (TMEM27). Diabetologia. doi:10.1007/ s00125-012-2605-2

2. Thorel F, Nepote V, Avril I et al (2010) Conversion of adult pancreatic alpha-cells to beta-cells after extreme beta-cell loss. Nature 464:1149-1154

3. Meier JJ, Bhushan A, Butler AE, Rizza RA, Butler PC (2005) Sustained beta cell apoptosis in patients with long-standing type 1 diabetes: indirect evidence for islet regeneration? Diabetologia 48:2221-2228

4. Meier JJ, Menge BA, Breuer TG et al (2009) Functional assessment of pancreatic beta-cell area in humans. Diabetes 58:1595-1603

5. Mu J, Woods J, Zhou YP et al (2006) Chronic inhibition of dipeptidyl peptidase- 4 with a sitagliptin analog preserves pancreatic beta-cell mass and function in a rodent model of type 2 diabetes. Diabetes 55:1695-1704

6. Veluthakal R, Harris P (2010) In vivo beta-cell imaging with VMAT 2 ligands - current state-of-the-art and future perspective. Curr Pharm Des 16:1568-1581

7. Andralojc K, Srinivas M, Brom M et al (2012) Obstacles on the way to the clinical visualisation of beta cells: looking for the Aeneas of molecular imaging to navigate between Scylla and Charybdis. Diabetologia 55:1247-1257

8. Virostko J, Henske J, Vinet L et al (2011) Multimodal image coregistration and inducible selective cell ablation to evaluate imaging ligands. Proc Natl Acad Sci U S A 108:20719-20724

9. Reiner T, Kohler RH, Liew CW et al (2010) Near-infrared fluorescent probe for imaging of pancreatic beta cells. Bioconjug Chem 21:1362-1368

10. Reiner T, Thurber G, Gaglia J et al (2011) Accurate measurement of pancreatic islet beta-cell mass using a second-generation fluorescent exendin-4 analog. Proc Natl Acad Sci U S A 108:12815-12820

11. Brogren CH, Hirsch F, Wood P, Druet P, Poussier P (1986) Production and characterization of a monoclonal islet cell surface autoantibody from the BB rat. Diabetologia 29:330-333

12. Moore A, Bonner-Weir S, Weissleder R (2001) Noninvasive in vivo measurement of beta-cell mass in mouse model of diabetes. Diabetes 50:2231-2236

13. Ueberberg S, Meier JJ, Waengler C et al (2009) Generation of novel single-chain antibodies by phage-display technology to direct imaging agents highly selective to pancreatic beta- or alpha-cells in vivo. Diabetes 58:2324-2334

14. Akpinar P, Kuwajima S, Krutzfeldt J, Stoffel M (2005) Tmem27: a cleaved and shed plasma membrane protein that stimulates pancreatic beta cell proliferation. Cell Metab 2:385-397

15. Esterhazy D, Stutzer I, Wang H et al (2011) Bace2 is a beta cellenriched protease that regulates pancreatic beta cell function and mass. Cell Metab 14:365-377

16. Flamez D, Roland I, Berton A et al (2010) A genomic-based approach identifies FXYD domain containing ion transport regulator 2 (FXYD2) $\gamma$ a as a pancreatic beta cell-specific biomarker. Diabetologia 53:1372-1383

17. Hald J, Galbo T, Rescan C et al (2012) Pancreatic islet and progenitor cell surface markers with cell sorting potential. Diabetologia 55:154-165

18. Sharpe J, Ahlgren U, Perry P et al (2002) Optical projection tomography as a tool for 3D microscopy and gene expression studies. Science 296:541-545 